\title{
CHEMICAL AND DYNAMICAL HISTORY OF THE MILKY WAY
}

\author{
I.F.BIKMAEV \\ Special Astrophysical Observatory, Nizhnij Arkhyz, \\ Karachai-Circassian Republic, Russia, 357147 \\ AND \\ L.I.MASHONKINA, N.A.SAKHIBULLIN, V.V.SHIMANSKIJ AND \\ N.SHIMANSKAYA \\ Department of Astronomy, Kazan State University, \\ Lenin Str.,18, Kazan, Russia, 420008
}

\section{Introduction}

F-G dwarf stars of different metallicity are used as tracers of chemical and dynamical history of the Galaxy. Observed ratios of element abundances " $\mathrm{X} / \mathrm{Fe}]$ versus $[\mathrm{Fe} / \mathrm{H}]$ " (where " $\mathrm{X}$ " is a some of chemical element) are compared with theoretical ones and allow us to make the choice between models (or parameters of theoretical models) of the Galaxy chemical evolution, nucleosynthesis and processes of star formation. Our experience has shown (Bikmaev, 1991, 1994a,b; Bikmaev et al., 1990) that in some cases classical approaches in abundance determination are affected by methodical uncertainties and the spread in $[\mathrm{X} / \mathrm{Fe}]$ ratios may be large even if high quality spectra used.

Our recent investigation based on: 1) high resolution spectra of 60 stars obtained on the 6-m telescope (Bikmaev, 1987) and carefully selected homogeneous list of published equivalent widths of spectral lines, 2) Kurucz's (1979) grid of model atmospheres and his WIDTH6 program, 3) temperature scale of the Infrared Flux Method and use of infrared (V-K) or red (R-I) color indices for effective temperature determination, 4) trigonometric parallaxes and the "temperature-mass-luminosity" dependence for obtaining surface gravity, 5) non-LTE computations for FeI, BaII, NaI and MgI lines, 6) use of FeII lines for metallicity determinations. 


\section{Results of non-LTE computations}

Deviations from LTE for FeI lines strongly depend on a number of levels in the FeI model atom and an adapted value of cross-section of collisions with hydrogen atoms (Bikmaev et al., 1990, Gehren et al., 1991, Steenbock, 1991). It was found (Bikmaev, 1994b) that problems in FeI-FeII ionization balance, probably, lie out of the classical methods of non-LTE computations using of homogeneous model atmospheres. Therefore, we used FeII lines for iron abundance determination taking into account that they show no any deviations from LTE (Steenbock, 1991) and are insensitive to possible effects of temperature inhomogeneities in the atmospheres of real stars (see Fig.2 of Bikmaev,1994a). Metallicity scale based on the FeII lines has changed for extremely metal-poor stars: a value of $[\mathrm{Fe} / \mathrm{H}]=-2.5$ in the classical case when $\mathrm{FeI}$ lines used, corresponds to $[\mathrm{Fe} / \mathrm{H}]=-2.0$ in our scale (Bikmaev et al., 1990), Figs. 1-8.

$\mathrm{MgI}$ lines (excluding $4571 \AA$ and Mgb lines) show small deviations from LTE (0.04-0.12 dex) depending on adapted value of cross-section of collisions with hydrogen atoms. So we used LTE abundances in this study.

It was found (Mashonkina et al., 1993) that only $6154 \AA$ and 6160 $\AA$ sodium lines have small (0.05-0.10 dex) non-LTE corrections. All other lines show large negative non-LTE effects (up to -0.3-0.5 dex for $\mathrm{NaD}$ and infrared lines in the case of metal-poor stars where they are only measurable in the spectra, see Fig.7).

Note that we used an estimate of the value of positive non-LTE correction for resonance aluminum lines (Fig. 8) from the paper of Gehren et al., 1991. Taking into account large non-LTE effects with a different sign for resonance sodium and aluminum lines solves the problem of a discrepancy in $[\mathrm{Na} / \mathrm{Mg}]$ and $[\mathrm{Al} / \mathrm{Mg}]$ ratios found for halo stars (Zhao and Magain, 1990).

All 4 measurable BaII lines show their own range of non-LTE corrections (0.05-0.20 dex) with a change of sign when metallicity and effective temperature change (Mashonkina and Bikmaev, 1994). Therefore we were forced to compute individual non-LTE corrections for all 140 stars in our sample. We used in this study only 4554 and $5853 \AA$ lines showing smallest deviations from LTE. We did not take into account the hyperfine structure in $4554 \AA$ line, trying to find this effect from difference in abundances obtained by using both these lines (Fig. 3 ).

\section{Results and discussions}

1) Fig.1 shows that distribution of subdwarfs by metallicity is a nonmonotonic function and reflects the existence of three Galactic populations (halo, thick disk and disk) having their own ranges of metallicity. 
2) There are the sharp boundaries in kinematic characteristics of halo, thick disk and disk stars at the metallicity values $[\mathrm{Fe} / \mathrm{H}]=-0.9$ and -0.4 (Figs. 2, 4, 10). Note that at these Figures each dot represents one star. The data of the ordinates in Figs. 2, 4 are taken from the paper of Carney et al. (1990) and from catalogues of Marsakov and Shevelev (1988) and of Shevelev (1988), obtained from Soviet Astronomical Data Center (Moscow). All data for Fig. 10 were taken from the paper of Edvardsson et al., 1993.

These kinematical boundaries occur at the positions of two main minima in Fig. 1 and support the reality of non-monotonic metallicity distribution. Halo, thick disk and disk have their own dynamical history.

3) Fig. 5-8 show that abundance ratios $[\mathrm{O} / \mathrm{Fe}],[\mathrm{Eu} / \mathrm{Fe}],[\mathrm{Na} / \mathrm{Mg}]$, $[\mathrm{Al} / \mathrm{Mg}]$ change their behavior near the metallicity value $[\mathrm{Fe} / \mathrm{H}]=-0.9$ which corresponds to kinematical boundary between halo and thick disk.

4) $[\mathrm{Ba} / \mathrm{Fe}]$ ratio determined from resonance $4554 \AA$ line shows nonlinear dependence on $[\mathrm{Fe} / \mathrm{H}]$ with breaks at $[\mathrm{Fe} / \mathrm{H}]=-0.9-1.0$ and $-0.4-0.5$ where changes in kinematics and in metallicity distribution are occurred (Fig.3). An excess in barium abundances obtained from $4554 \AA$ line relative of those derived from $5853 \AA$ line is the manifestation of influence the hyperfine structure to equivalent widths of resonance line as suggested by Cowley and Frey, 1989. According these authors part of barium isotopes are produced mostly during the r-process. Therefore, we, probably, have the indications of active phases in star formation and nucleosynthesis corresponding to periods of thick disk and disk stars formation. Decrease of $[\mathrm{Ba} / \mathrm{Fe}]$ ratio inside of disk and thick disk with increase of $[\mathrm{Fe} / \mathrm{H}]$ can be explained as interruption of active phases and "quiet" chemical evolution on the Galaxy space scales. Note that the same behavior as for barium $4554 \AA$ line, can, probably, be seen in the data of Andersson and Edvardsson (1994) for carbon (Fig. 9) and data of Edvardsson et al. (1993) for zirconium. Naturally, this kind of result should be confirmed by additional investigations.

\section{Conclusions}

Our results support and develop the hypothesis of the existence an active phases during the Galaxy evolution (Suchkov, 1977, 1981, Marsakov and Suchkov, 1976, 1977, 1978). Active phases correspond to separate formation of three Galaxy stellar populations - halo, thick disk and disk, having their own chemical and dynamical history. Theoretical models should take into account the significant changes of chemical and dynamical properties at the boundaries (by metallicity) of each stellar population. 


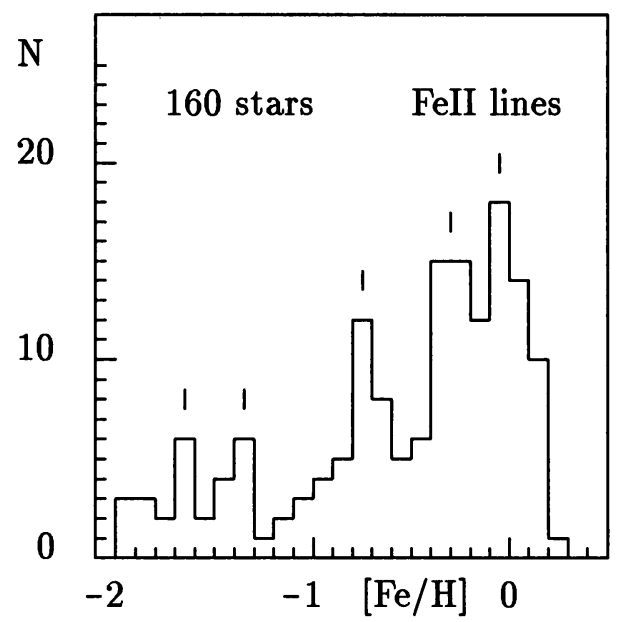

Fig. 1. Metallicity distribution of the sample of stars.

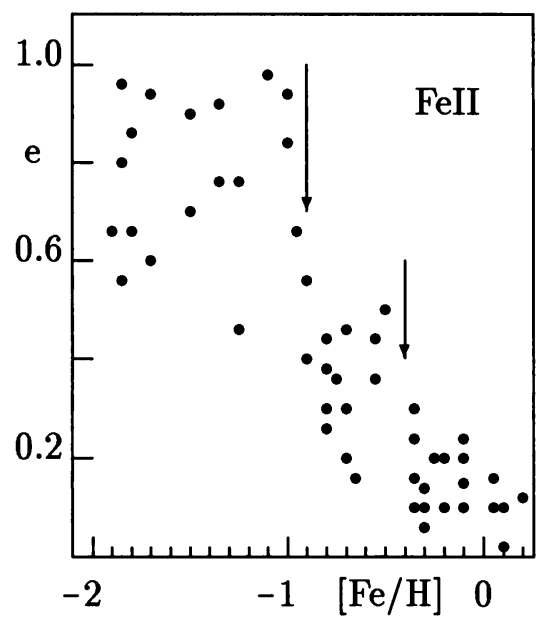

Fig. 2. Eccentricity versus metallicity.

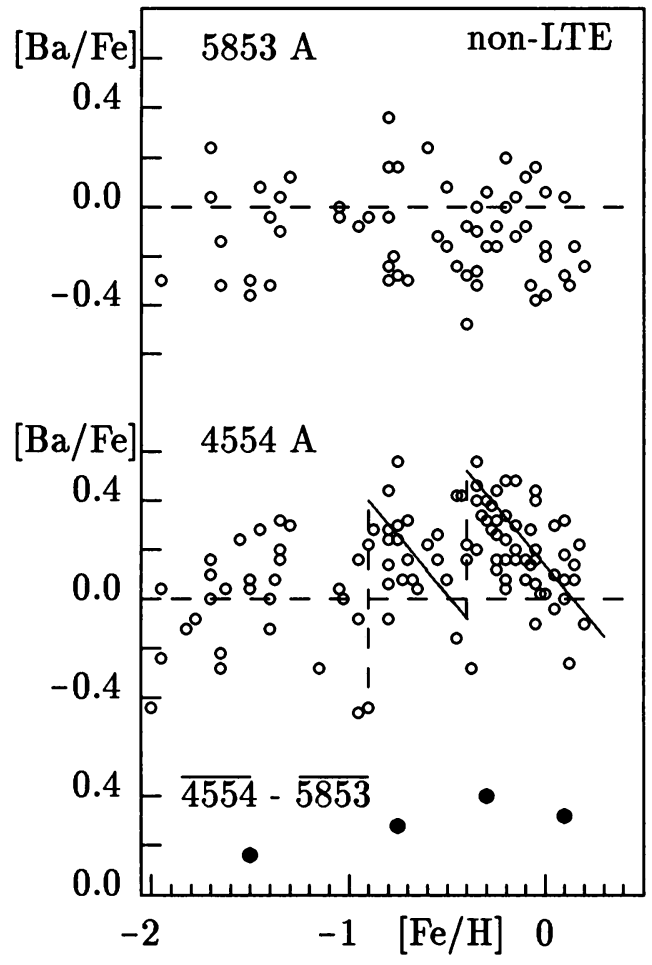

Fig. 3. $[\mathrm{Ba} / \mathrm{Fe}]$ versus $[\mathrm{Fe} / \mathrm{H}]$.

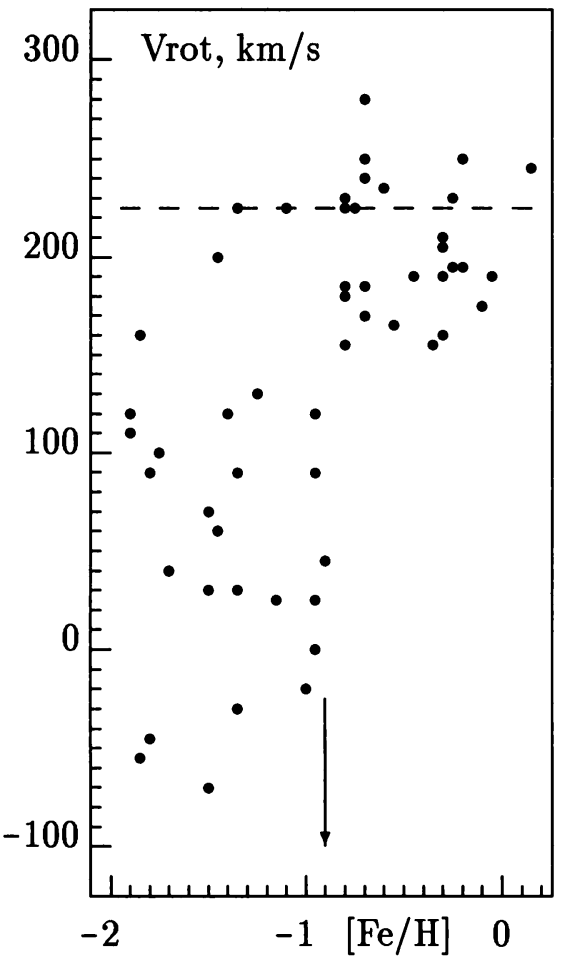

Fig. 4. Angular momentum versus metallicity. 
$[\mathrm{O} / \mathrm{Fe}]$

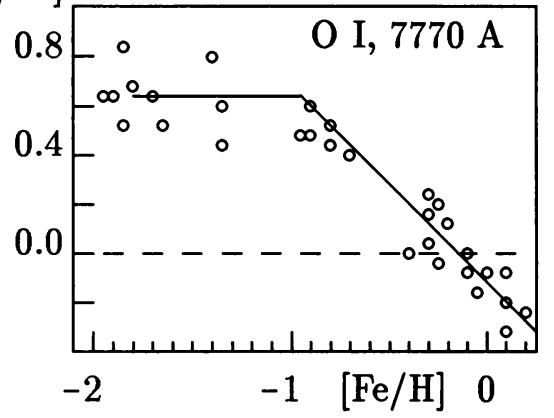

Fig. 5. $[\mathrm{O} / \mathrm{Fe}]$ versus $[\mathrm{Fe} / \mathrm{H}]$.
$[\mathrm{Eu} / \mathrm{Fe}]$

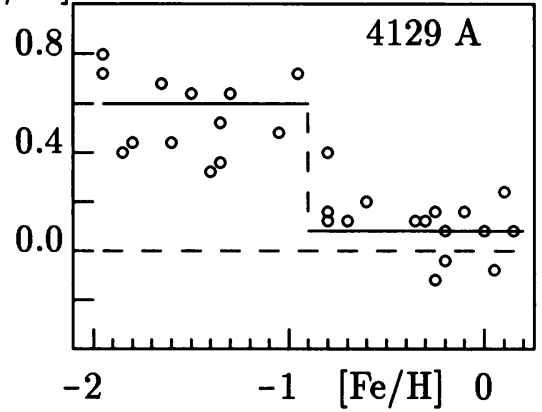

Fig. 6. $[\mathrm{Eu} / \mathrm{Fe}]$ versus $[\mathrm{Fe} / \mathrm{H}]$.
$[\mathrm{Na} / \mathrm{Mg}]$

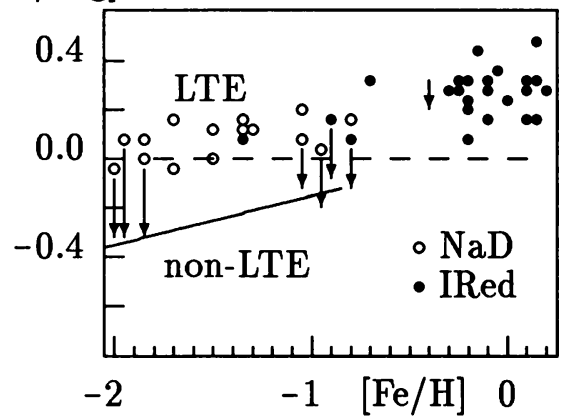

Fig. 7. $[\mathrm{Na} / \mathrm{Mg}]$ versus $[\mathrm{Fe} / \mathrm{H}]$.
$[\mathrm{Al} / \mathrm{Mg}]$

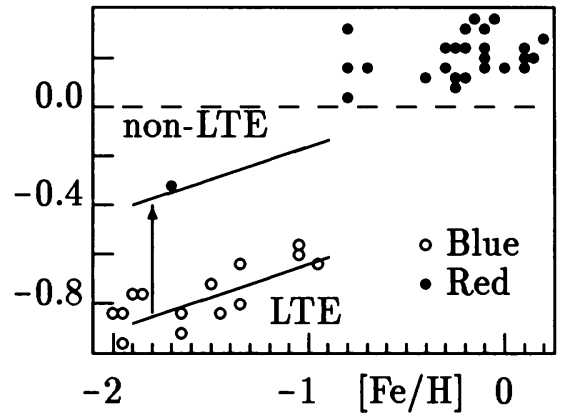

Fig. 8. $[\mathrm{Al} / \mathrm{Mg}]$ versus $[\mathrm{Fe} / \mathrm{H}]$.

$[\mathrm{C} / \mathrm{Fe}]$
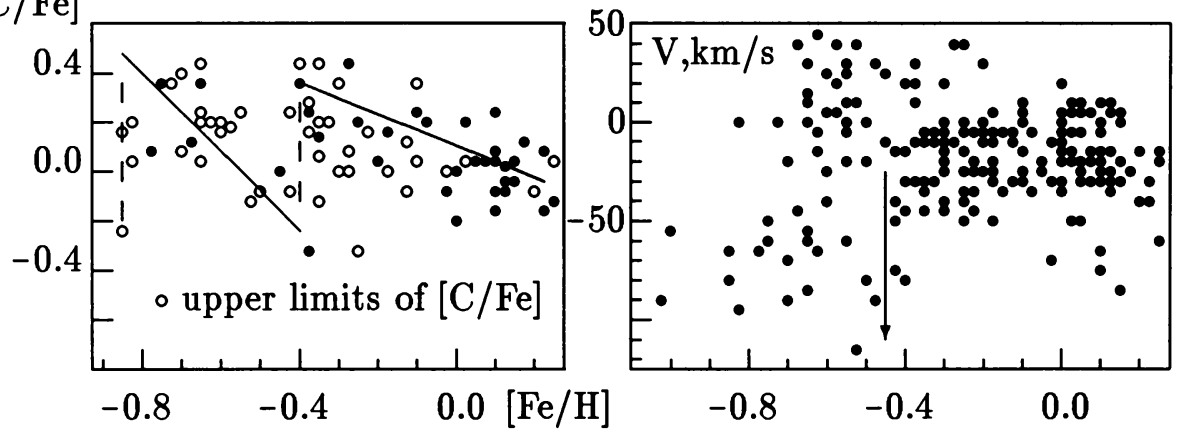

Fig. 9. $[\mathrm{C} / \mathrm{Fe}]$ versus $[\mathrm{Fe} / \mathrm{H}]$.

Data are taken from paper of Andersson and Edvardsson (1994).

Fig. 10. V-component of space motions relative to the Sun $(\mathrm{Vo}=226 \mathrm{~km} / \mathrm{s})$ versus $[\mathrm{Fe} / \mathrm{H}]$. Data are taken from paper of Edvardsson et al. (1993). 


\section{Acknowledgements}

This study was partly supported by grant of AAS to I.F.Bikmaev. This support is greatfully acknowledged. I.F.Bikmaev is also grateful to Drs. V.G.Klochkova and V.E.Panchuk for help during observational runs on the 6 -m telescope and the IAU for travel grant allowing presentation of this report.

\section{References}

Andersson, H. and Edvardsson, B. (1994) Astr.Astroph., 290, 590-598

Bikmaev, I.F. (1987) Bull. of the Special Astrophys. Obs.-North Caucasus, Allerton Press Translation Series, 25, 3-12

Bikmaev, I.F. (1991) PhD Thesis, Nizhnij Arkhyz

Bikmaev, I.F. (1994a) in: ASP Conference Series, Physics of the Gaseous and Stellar Disks of the Galaxy, ed.I.R.King, 66, 187-191

Bikmaev, I.F. (1994b) in: IAU XXIInd General Assembly 1994 Poster Abstracts, ed. H. van Woerden, p.231

Bikmaev, I.F., Bobritskij, S.S. and Sakhibullin, N.A. (1990) Sov. Astr. Lett., 16, 91-95

Carney, B.W., Aguilar, L., Latham, D.W. and Laird, J.B. (1990) Astron.J., 99, 201-220

Cowley, Ch.R. and Frey, M. (1989) Astroph.J., 346, 1030-1034

Edvardsson, B., Andersen, J., Gustafsson, B., Lambert, D.L., Nissen, P.E. and Tomkin, J. (1993) Astr.Astroph., 275, 101-152

Gehren, T., Reile, C. and Steenbock, W. (1991) in: Stellar atmospheres: Beyond classical models, eds. L.Crivellari,I.Hybeny and D.G.Hummer, Kluwer, Dordrecht, p.387

Kurucz, R.L. (1979) Ap.J.S.Ser., 40, 1-340

Marsakov, V.A. and Shevelev, Yu.G. (1988) Bulletin d'Information du CDS, 35, 129-130

Marsakov, V.A. and Suchkov, A.A. (1976) Sov.Astron.Lett., 2, 381-385

Marsakov, V.A. and Suchkov, A.A. (1977) Sov.Astr., 54, 1232-1240

Marsakov, V.A. and Suchkov, A.A. (1978) Sov.Astr., 55, 472-481

Mashonkina, L.I., Sakhibullin, N.A. and Shimanskij, V.V. (1993) Sov.Astron., 70, 372380

Mashonkina, L.I. and Bikmaev, I.F. (1994) submited to Sov.Astr.Lett.

Shevelev, Yu.G. (1988) Bulletin d'Information du CDS, 35, 135-136

Steenbock, W. (1991) private communication

Suchkov, A.A. (1977) Sov.Astr.Lett., 3, 165-169

Suchkov, A.A. (1981) Aph.Sp.Sci., 77, 3-22

Zhao, G. and Magain, P. (1990), Astr.Astroph., 238, 242-248 